\title{
Relations between a computerized shopping task and cognitive tests in a group of persons diagnosed with schizophrenia compared with healthy controls
}

\author{
FRANK LARøI, ${ }^{1,2}$ JENNIFER CANLAIRE, ${ }^{1}$ HAITHAM MOURAD, ${ }^{2}$ AND \\ MARTIAL VAN DER LINDEN ${ }^{1,3}$ \\ ${ }^{1}$ Cognitive Psychopathology Unit, University of Liège, Liège, Belgium \\ ${ }^{2}$ Intercommunale de Soins Spécialisés de Liège (Mental Health Sector), Liège, Belgium \\ ${ }^{3}$ Cognitive Psychopathology and Neuropsychology Unit, University of Geneva, Geneva, Switzerland
}

(Received January 20, 2009; Final Revision October 2, 2009; ACCEPTED October 5, 2009)

\begin{abstract}
Cognitive deficits are clearly associated with poor everyday life functioning in persons diagnosed with schizophrenia. However, previous studies have primarily used questionnaires to assess everyday life functioning. We developed a computerized real-life activity task (shopping task), where participants are required to shop for a list of seven grocery store items. Thirty individuals diagnosed with schizophrenia and 30 healthy controls were administered an extensive battery of cognitive tests and the computerized shopping task. Performances on the computerized shopping task significantly differentiated patients and healthy controls for several variables. Moreover, performance on the shopping task was significantly correlated with verbal episodic memory, cognitive flexibility, planning, processing speed, and inhibition. Finally, performance on the computerized shopping task was significantly correlated with various clinical variables and with a global measure of social functioning. These findings suggest that the computerized task used in the present study provides an indication of the level of everyday life functioning and cognitive functioning of persons diagnosed with schizophrenia, and, therefore, may be viewed as a valuable instrument in both an evaluation and remediation context. (JINS, 2010, 16, 180-189.)
\end{abstract}

Keywords: Everyday life activities, Neuropsychology, Ecological validity, Neurocognition, Social functioning, Computerized

\section{INTRODUCTION}

Several studies have shown that cognitive deficits in individuals diagnosed with schizophrenia are associated with various aspects of everyday life such as social adjustment, work functioning, independent life, and interpersonal relations (Bowie, Reichenberg, Patterson, Heaton, \& Harvey, 2006; Green, 1996; Green, Kern, Braff, \& Mintz, 2000; McGurk \& Meltzer, 2000; Velligan, Bow-Thomas, Mahurin, Miller, \& Halgunseth, 2000). However, the majority of studies have examined everyday life functioning using questionnaires, which may not reflect the actual everyday lives of patients. Furthermore, results from questionnaires may be biased as self-reports or care-giver reports are often used

Correspondence and reprint requests to: Frank Larøi, Cognitive Psychopathology Unit, Department of Cognitive Sciences, University of Liège, Bd. du Rectorat (B33), B-4000 Liège, Belgium. E-mail: flaroi@ulg.ac.be to measure everyday life functioning. Self-report may be influenced by several variables such as patients' level of insight, psychopathology, and cognitive and emotional functioning. Similarly, care-giver reports are limited by the lack of persons available to report on patients' everyday functioning and by care-givers' own cognitive, emotional, and psychiatric functioning (McKibbin, Brekke, Sires, Jeste, \& Patterson, 2004).

An alternative to using questionnaires is to use performancebased measures of functional living skills, which involves directly observing patients while they perform everyday life activities. Such an approach provides both greater specificity regarding social functioning and more reliable and valid information concerning everyday life abilities. A growing number of studies have used performance-based measures of functional living skills in persons diagnosed with schizophrenia, which examine either a wide range of everyday activities (Patterson, Goldman, McKibbin, Hughs, \& Jeste, 
2001) or focus on a relatively specific activity such as medication management abilities (Kurtz, Baker, Pearlson, \& Astur, 2007), preparing a meal (Semkovska, Bédard, Godbout, Limoge, \& Stip, 2004), or shopping for items in a grocery store (Rempfer, Hamera, Brown, \& Cromwell, 2003). The majority of these measures are observation-based (Patterson et al., 2001; Rempfer et al., 2003; Semkovska et al., 2004), with the exception of the computerized task developed by Kurtz et al. (2007).

To date, two studies (Rempfer et al., 2003; Semkovska et al., 2004) have used observation-based measures that specifically involve a shopping task in persons diagnosed with schizophrenia. In Rempfer et al. (2003), 73 individuals with a schizophrenia diagnosis completed a battery of cognitive tests and then completed the Test of Grocery Shopping Skills (TOGSS; Hamera \& Brown, 2000; Hamera, Brown, Rempfer, \& Davis, 2002). The cognitive tests consisted of conventional tests assessing functions such as attention, memory, and executive abilities. Concerning the TOGSS, participants were required to shop for a list of 10 grocery store items, selecting the correct size and lowest possible price for each item on the list. Three scores were calculated: accuracy (is the selected item the correct item, the correct size, and the lowest price?), redundancy (number of excess aisles of the grocery store divided by the total number of aisles in the grocery store), and time taken to complete the task. Results revealed significant correlations between the TOGSS (accuracy and redundancy) and tests measuring executive and attentional functions. In particular, accuracy was significantly correlated with processing speed and sustained attention, verbal memory, and perseverative errors (based on the Wisconsin Card Sorting Task; WCST; Heaton, Chelune, Talley, Kay, \& Curtiss, 1993). More redundancy during the task was related to poor performance on various executive tests and verbal memory. Finally, time was related to a measure of verbal memory. It is important to note that correlations were weak to moderate, and that no control group or clinical measures were included in this study.

More recently, Semkovska et al. (2004) examined relations between various everyday tasks and a battery of conventional cognitive tests assessing memory and executive functioning in a group of 28 patients diagnosed with schizophrenia and 27 healthy controls. The everyday task consisted of three different activities: choosing a menu, shopping for the ingredients, and cooking a meal. Results showed that when choosing the menu, patients with schizophrenia made more omissions compared with matched controls. During the shopping task, patients committed more sequencing errors and repetitions than controls. Finally, cooking the meal was clearly the most challenging as the two groups differed significantly on all the measures. None of the cognitive variables or performances on the everyday tasks correlated significantly with patients' duration of illness or length of hospitalization (a global functioning measure was not included in the study). Furthermore, certain errors on the activities of daily living task correlated significantly with negative and positive symptoms, and with general psychopathology. However, specifically concerning the shopping task, there were no correlations between symptomatology and performance on this task. Finally, in general, performances on the daily living activities correlated significantly with performances on executive tests (especially those sensitive to shifting and sequencing abilities), but not with memory tests. In particular, performance on the shopping task was only associated with one cognitive test, namely, scores on the Picture Arrangement subtest of the Wechsler Adult Intelligence Scale-Revised (WAIS-R; Wechsler, 1981).

These studies confirm the close relationship between cognitive deficits and poor performance on simulations of everyday life activities. Furthermore, in that they include observationbased measures, these studies provide evidence of this relation while avoiding problems (mentioned above) inherent in questionnaire-based assessment of social functioning. Indeed, the obvious advantage of observation-based functional assessments is that the actual everyday tasks themselves are being assessed. However, there are also several limits associated with observation-based measures. Carrying out observation-based functional assessments in a clinical setting may be difficult in both practical terms (e.g., finding the time and opportunity to observe patients in a grocery store) and in terms of human resources (e.g., having the available personnel that can leave the clinical setting to perform this type of assessment). Furthermore, a series of variables cannot be controlled (e.g., variations in the number of shoppers, the amount of noise, and other distractions). Also, it is difficult to obtain precise measures of performances when conducting qualitative observations; moreover, only a limited number of variables can be encoded and calculated as there are constraints as to how much the observer can note down. One way of avoiding many of these limits is to develop a computerized (or virtual reality) version of the everyday life activity where the environment is the same for all participants and where a large number of variables can be calculated in a quantitative and precise manner. It is important to underline, however, that the aim is not to replace observation-based functional assessments, but rather to propose an alternative, that is, by computerizing (certain) everyday activities, which, furthermore, takes into account some of the limits of observation-based functional assessment.

In the present study, we wished to examine relations between a real-life computerized task (shopping task) and cognitive tests in a group of individuals diagnosed with schizophrenia and in a group of healthy controls. The first goal was to compare the two groups in terms of their performances on conventional cognitive tests, and thereafter, to compare them in terms of their performances on the computerized shopping task. Relations between cognitive tests and the computerized shopping task in the patient group were also examined. Finally, a series of clinical and demographic variables were related to performance on the computerized shopping task.

It was hypothesized that the performance on the computerized shopping task would significantly differentiate the group of patients with schizophrenia and the group of healthy controls. Furthermore, based on previous studies, we hypothesized significant correlations between patients' performance on 
the computerized shopping task and measures of cognitive testing, in particular with tests assessing memory, executive functions, and processing speed.

\section{METHODS}

\section{Participants}

Thirty persons diagnosed with schizophrenia according to DSM-IV criteria (American Psychiatric Association, 1994) were included. Exclusion criteria consisted of the presence of neurological and/or other psychiatric disorders and electroconvulsive therapy. Seventeen patients were hospitalized and 13 were nonhospitalized. Mean chlorpromazine (CPZ) equivalents for the patient group was 365 , and there were no significant group differences ( $t$ tests) between hospitalized and nonhospitalized patients in terms of mean $\mathrm{CPZ}$ equivalents $(t(28)=1.08 ; p=.28)$. Thirty controls were also included who were matched as closely as possible according to sex, age, and educational level. The controls did not have any psychiatric or neurological disorders nor was there any family history of psychiatric or neurological disorders. All participants provided written informed consent, and the project was approved by the local ethics committee. Participant characteristics are presented in Table 1. Because both groups differed significantly in terms of years of education and pre-morbid IQ, these two variables were covaried in subsequent analyses.

\section{Measures}

\section{Clinical measures}

All patients were evaluated with the French version of the Global Assessment of Functioning (GAF; American Psychiatric Association, 1994) Scale and the French version of the Positive and Negative Syndrome Scale (PANSS; Kay, Opler, $\&$ Fisbein, 1987). The GAF is a commonly used measure to assess the social, occupational, and psychological functioning of adults. Scores vary between 0 and 100, with low scores indicating low functioning. Studies examining the psychometric properties of the GAF reveal adequate levels of levels of reliability and validity (Hilsenroth et al., 2000; Jones, Thornicroft, Coffey, \& Dunn, 1995). In addition to the PANSS Total score, separate scores from the PANSS were calculated for the Positive symptom sub-scale (assessing the presence of symptoms such as hallucinations and delusions), the Negative symptom sub-scale (evaluating signs such as blunted affect and emotional withdrawal), and the General psychopathology sub-scale (which includes items pertaining to several symptoms such as depression, anxiety, and somatic concern). All clinical ratings were carried out by an experienced clinical psychiatrist. The clinical ratings were made during the same period, that is, between the computerized shopping task and the cognitive assessment.

\section{Cognitive measures}

Participants were also assessed with an extensive battery of well-known, standardized cognitive tests measuring:

1. Pre-morbid IQ: National Adult Reading Test (NART; Mackinnon \& Mulligan, 2005; Nelson, 1982).

2. Verbal episodic memory: California Verbal Learning Test (CVLT; Delis, Freeland, Kramer, \& Kaplan, 1988; Poitenaud, Deweer, Kalafat, \& Van der Linden, 2007).

3. Visual episodic memory: Face recognition (Wechsler, 2001).

4. Selective attention: The D2 Test of Attention (Brickenkamp, 1966).

5. Executive functions: Phonologic and semantic verbal fluency (Cardebat, Doyon, Puel, Goulet, \& Joannette, 1990), Zoo map (from The Behavioral Assessment of the Dysexecutive Syndrome test battery; Wilson, Alderman, Burgess, Emslie, \& Evans, 1996), Trail Making Test (Army Individual Test Battery, 1944; time on Part B minus time on Part A), Stroop Time interference (Golden, 1978).

Table 1. Demographic characteristics of participants

\begin{tabular}{|c|c|c|c|c|}
\hline & \multicolumn{2}{|c|}{ Patients $(n=30)$} & \multicolumn{2}{|c|}{ Healthy controls $(n=30)$} \\
\hline & Min-max & Mean $(S D)$ & Min-max & Mean $(S D)$ \\
\hline Age & $19-50$ & $31.13(8.32)$ & $19-51$ & $30.53(9.0)$ \\
\hline Education (years) & $9-17$ & $11.97(1.61)$ & $9-19$ & $13.63(2.31)^{* *}$ \\
\hline Pre-morbid IQ (NART) & & $105.68(9.2)$ & & $111.55(9.1)^{*}$ \\
\hline Sex (female/male) & $5 / 25$ & & $5 / 25$ & \\
\hline Inpatients/outpatients & $17 / 13$ & & & \\
\hline Number of hospitalizations & $1-18$ & $5.8(3.95)$ & & \\
\hline Duration of illness (years) & $1-23$ & $8.5(6.82)$ & & \\
\hline GAF & $29-84$ & $59.30(16.40)$ & & \\
\hline PANSS Total & $34-108$ & $66.23(21.77)$ & & \\
\hline Positive sub-scale & $7-24$ & $14.47(5.73)$ & & \\
\hline Negative sub-scale & $7-36$ & $19.93(7.75)$ & & \\
\hline General psychopathology sub-scale & $16-56$ & $31.57(11.08)$ & & \\
\hline
\end{tabular}

Note. NART $=$ National Adult Reading Test GAF = Global Assessment of Functioning Scale; PANSS = Positive and Negative Syndrome Scale.

$* p<.05 ; * *<<.01$. 
6. Working memory: Digit span (forward, backward, letternumber sequencing; Wechsler, 2001).

7. Processing speed: Stroop Color Naming Time (Golden, 1978), WAIS Processing speed index (scores on the Digit symbol and Symbol search; Wechsler, 2000).

\section{Computerized shopping task ${ }^{1}$}

All participants completed the computerized shopping task, which was programmed in C\# and DirectX 9. The time between administration of the cognitive battery and the computerized shopping task was on average 3.48 days for the whole group ( 4.13 days for the patients and 2.83 days for the healthy controls). All participants first completed a learning phase and, thereafter, completed the shopping task.

Learning phase-The main goal of this learning phase was to systematically familiarize all the participants with the basic actions and functions that are required in the shopping task, and to do so successfully (i.e., without committing any errors). The learning phase was based on the principles of errorless learning, which has proven particularly effective in patients diagnosed with schizophrenia (cf. Kern, Green, Mintz, \& Liberman, 2003; Kern, Liberman, Kopelowicz, Mintz, \& Green, 2002; O'Carroll, Russell, Lawrie, \& Johnstone, 1999). In this context, the learning phase consisted of carrying out task-relevant actions (i.e., those that are required during the shopping task) in a progressive and error-limiting manner. That is, to begin with, participants were required to perform very simple actions followed by gradually more demanding and complex actions. Furthermore, only when participants performed each action successfully, did they proceed to the next level of the learning phase. Instructions were given both visually on the screen and orally (by means of a computerized voice) throughout the learning phase. If the participant committed an error at any time during the learning phase, the error was registered, the participant was alerted of this error, the instructions were repeated, and the participant was asked to continue until the action was performed without committing an error.

To begin with, the various functions of the gamepad (see Figure 1) were introduced to the participant. This consisted of explaining the functions on the left-hand side of the gamepad, that is, for movement of the computerized person (forward, backward, left, and right) and those on the right-hand side of the gamepad (i.e., buttons A and B), which allow participants to perform various actions (open doors, view lists, open choice-menus, etc.). Specifically, participants were first asked to move the computerized person forward, backward, left, and right. Participants were then required to go to various boxes and to open and close them. Following this, participants were asked to go to a box, take an object, and put the object in a specific place. Subsequently, the task entailed entering a house, going toward a bookshelf and

${ }^{1}$ For those interested in obtaining the shopping task (free of charge), please contact the first author.

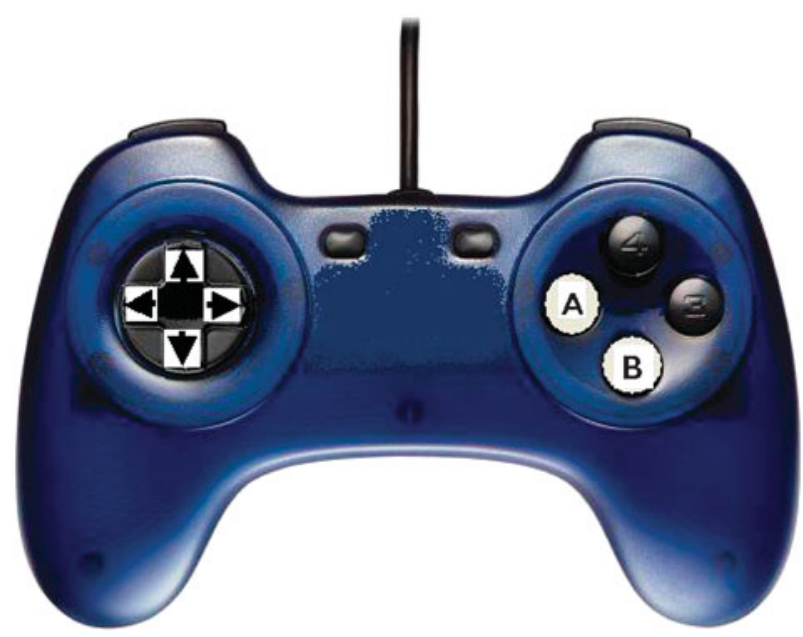

Fig. 1. The gamepad used during the learning phase and the shopping task.

choosing a specific book. The final phase consisted of asking participants to perform a task (paint a room) where they were asked to read a list of the required items (paint, gloves, and paintbrush), to go to the shelf and to choose the correct items from the shelf.

Shopping task-After the learning phase, participants were told that the shopping task was to begin. The same gamepad used in the learning phase was also used for the shopping task. The instructions were given orally by a computerized voice and were presented visually on the screen. When the instructions were understood, the participant was invited to press a button to view the shopping list consisting of seven items, representing different categories (e.g., beverages, meat, fruits, and vegetables). The items were: "Cool Fresh" lemonade fizzy drink, chicken brochettes, "Crock" biscuits, "Lonnux" washing liquid, "Télé Revue" magazine, green apples, and "Marque $\mathrm{n}^{\circ} 1^{\text {" long grain rice. All items }}$ were the same for each participant, and there was no time limit. The participant pressed a button to start the task. During the task, by pressing button A, participants could perform actions including having a close-up of the aisle, putting an item into the shopping cart, or putting an article back into the aisle. Furthermore, by pressing button B, participants could consult the list of items and the contents of the shopping cart. Movement of the computerized person was done by using the left-hand side of the gamepad. Music was played in the background throughout the shopping task, which consisted of light, Vivaldi-type, classical music. A certain number of distracters were also provided, which included both visual (non-pertinent articles on sale and the presence of other shoppers) and auditory (loud-speaker announcements) distracters. In particular, this included three people randomly moving around the grocery store, three people standing still in front of an aisle, seven stands with (non-pertinent) items on sale, and six different loud-speaker announcements (announcing non-pertinent items for sale) that were presented randomly throughout the shopping task. The grocery store consisted of seven (double-sided) aisles in 
the center of the grocery store and included the following aisles: stationary, cleaning products, washing powder and toilette paper, perfume and hygienic products, coffee and tea, chocolate and biscuits, cereals and jellies, beverages, wine, meat and fish tins, fruit and vegetable tins, condiments and sauces, pasta and rice, and products on sale. There were also 11 aisles along the wall of the grocery store and included the following aisles: fruits and vegetables, bakery, delicatessen, cheese, meat, dairy products, and frozen food. There were also six stands containing (non-pertinent) items on sale that were located in the corners of the grocery store and at the ends of certain aisles. When the participant approaches the till, a message asking if they are ready to pay for the items appears. If the participant answers "no" then $\mathrm{s} / \mathrm{he}$ returns to the grocery store, if the participant replies "yes", then the task is terminated. The following variables were calculated for this task: total time to complete the shopping task (in seconds), number of correct items, number of intrusions (non-target items), number of corrected errors, aisle redundancy (number of times in the same grocery aisle), number of times in a non-pertinent aisle, number of times a non-pertinent aisle was visualized, number of times a participant consulted the shopping list, time spent consulting the shopping list, and number of times a participant consulted the shopping cart.

All participants were also asked to indicate the frequency that they go to the grocery store to buy food and the frequency that they play video games, both based on a 5-point Likert scale (never; once a month; once a week; 2 to 3 times a week; everyday). Based on independent $t$ tests, the two groups did not differ from each other both for frequency of shopping and frequency of playing video games.

\section{RESULTS}

Compared with healthy controls, patients had significantly poorer performances on tests assessing verbal fluency, verbal episodic memory, cognitive flexibility, planning, processing speed, and inhibition (Table 2). Because both groups differed significantly in terms of years of education and premorbid IQ, the same analyses were carried while covarying for these two variables. This revealed that significant group differences remained significant.

Patients and healthy controls were then compared concerning their performances on the computerized shopping task (Table 3). These analyses revealed that performances on the computerized shopping task significantly differentiated patients and healthy controls for: total time to complete the task, number of correct articles chosen, aisle redundancy (number of times in the same grocery aisle), number of times a non-pertinent aisle was visualized, number of times participants consulted the shopping list, and time participants spent consulting the shopping list. These same analyses were carried out while at the same time covarying for years of education and pre-morbid IQ, revealing that significant group differences remained significant.

The variables from the computerized shopping task that significantly differentiated between groups were then correlated with results from the cognitive tests (Table 4). A global cognitive test score was calculated, which consisted of converting the cognitive test scores to $Z$ scores (based on the results of the healthy control group) and then calculating the average $Z$ score for all cognitive test scores. The global cognitive test score (a low score indicates poor performance) correlated significantly with number of correct articles (i.e., less correct articles was associated with a more deficient global cognitive test score). Concerning correlations involving individual cognitive tests, there were significant correlations between total time to complete the task and cognitive flexibility [Trail Making Test (TMT) B-A]. Number of correct articles chosen was significantly correlated with a large number of cognitive measures, including verbal episodic memory (CVLT total correct 1-5), cognitive flexibility (semantic verbal fluency), planning (Zoo map), processing speed (Stroop Color Naming Time and WAIS Processing speed index), and inhibition (Stroop Time interference). Number of times a non-pertinent aisle was visualized was significantly correlated with cognitive

Table 2. Significant differences between the two groups for cognitive tests

\begin{tabular}{lcccc}
\hline \hline & \multicolumn{2}{c}{ Patients with schizophrenia } & & \multirow{2}{*}{ Healthy controls } \\
\cline { 2 - 3 } Variables & Mean $(S D)$ & & $t$ \\
\cline { 2 - 3 } Phonological verbal fluency & $17.73(5.93)$ & $25.03(8.0)$ & $-4.02^{* * *}$ \\
Semantic verbal fluency & $21.87(6.80)$ & $32.63(8.33)$ & $-5.48^{* * *}$ \\
CVLT total correct 1-5 & $39.50(11.23)$ & $60.97(8.46)$ & $-8.36^{* * *}$ \\
TMT B-A (Time) & $53.09(25.13)$ & $38.03(20.86)$ & $2.53^{*}$ \\
Zoo map & $2.93(3.42)$ & $5.40(2.61)$ & $-3.14^{* *}$ \\
Stroop Color Naming Time & $79.67(26.16)$ & $59.70(9.63)$ & $3.92^{* * *}$ \\
Stroop Time interference & $138.69(44.06)$ & $105.80(22.54)$ & $3.64^{* * *}$ \\
WAIS Processing speed index & $79.60(24.74)$ & $117.07(22.44)$ & $6.14^{* * *}$ \\
Letter-number sequencing & $4.37(1.07)$ & $5.20(1.19)$ & $-2.86^{* *}$ \\
\hline \hline
\end{tabular}

Note . CVLT $=$ California Verbal Learning Test; TMT $=$ Trail Making Test; WAIS = Wechsler Adult Intelligence Scale.

$* p<.05 ; * * p<.01 ; * * * p<.001$. 
Table 3. Significant differences between the two groups on the shopping task

\begin{tabular}{lccc}
\hline \hline & Patients with schizophrenia & & Healthy controls \\
\cline { 2 - 3 } Variables & Mean $(S D)$ & Mean $(S D)$ & $t$ \\
\hline Total time (in seconds) & $835.68(304.16)$ & $527.53(159.27)$ & $4.92^{* * *}$ \\
Number of correct articles & $5.96(1.56)$ & $6.67(0.61)$ & $-2.29^{*}$ \\
Aisle redundancy & $12.21(9.93)$ & $4.97(4.99)$ & $3.57^{* * *}$ \\
Number of times a non-pertinent & $2.00(1.72)$ & $0.77(1.07)$ & $3.33^{* *}$ \\
$\quad$ & & & \\
aisle was visualized & $10.96(4.60)$ & $8.63(3.26)$ & $2.26^{*}$ \\
Number of times consult list & $107.25(53.69)$ & $71.20(31.96)$ & $3.16^{* *}$ \\
Number of intrusions & $2.11(3.61)$ & $0.87(1.01)$ & 1.81 \\
Number of corrected errors & $0.64(0.84)$ & $0.53(0.90)$ & 0.49 \\
Number of times in a non-pertinent aisle & $2.71(1.36)$ & $2.53(1.74)$ & 0.45 \\
Number of times a participant consulted & $2.11(1.90)$ & $1.73(1.55)$ & 0.83 \\
$\quad$ & & & \\
\hline \hline
\end{tabular}

$* p<.05 ; * * p<.01 ; * * * p<.001$.

flexibility (semantic verbal fluency and TMT B-A) and processing speed (Stroop Color Naming Time). Finally, the number of times participants consulted the shopping list was significantly correlated with processing speed (Stroop Color Naming Time).

Concerning relations between demographic variables and performance on the computerized shopping task, the only significant correlations were between total time to complete the task and age $(0.44 ; p<.05)$, number of hospitalizations $(0.41 ; p<.05)$, and frequency playing video games $(-0.37$; $p<.05$ ). A comparison (independent $t$ tests) of hospitalized patients with nonhospitalized patients for the six variables that differentiated the patient group from the control group did not reveal any significant group differences.

Correlations between functioning level (GAF) and symptomatology (PANSS) with the performance on the computerized shopping task were also calculated (Table 4). This revealed that GAF scores significantly correlated with aisle redundancy $(0.54 ; p<.01)$ and the number of times participants consulted the shopping list $(0.41 ; p<.05)$. Correlations between GAF scores and the cognitive variables were also calculated. This revealed that only Stroop Time interference correlated with GAF scores $(-0.42 ; p<.05)$. Finally, the number of times participants consulted the list correlated significantly with the PANSS Total score $(-0.59 ; p<.01)$, Positive sub-scale $(-0.42 ; p<.05)$, Negative sub-scale $(-0.59 ; p<.01)$, and General psychopathology sub-scale $(-0.49 ; p<.05)$. The number of correct articles chosen correlated significantly with the PANSS Total score $(-0.51 ; p<.05)$, Positive sub-scale $(-0.45 ; p<.05)$, Negative sub-scale $(-0.46 ; p<.05)$ and General psychopathology sub-scale $(-0.46 ; p<.05)$. Aisle redundancy correlated significantly with the Negative sub-scale $(-0.46 ; p<.05)$.

Finally, the patient group was divided into those who had successfully collected all seven items and those who did not. This revealed that $53 \%$ of patients $(n=16)$ fell into the latter group. These two groups were then compared ( $t$ tests) in terms of the cognitive and clinical variables. No significant group differences were revealed.

\section{DISCUSSION}

Compared with healthy controls, patients with schizophrenia had significantly poorer performances on tests assessing verbal episodic memory, processing speed, and several executive functions. These results are generally in line with previous meta-analyses (Dickinson, Ramsey, \& Gold, 2007; Fioravanti, Carlone, Vitale, Cinti, \& Clare, 2005; Heinrichs \& Zaksanis, 1998).

Patients and healthy controls were significantly differentiated for the following variables of the computerized shopping task: total time to complete the task, number of correct articles chosen, aisle redundancy, number of times a nonpertinent aisle was visualized, number of times participants consulted the shopping list, and time participants spent consulting the shopping list. Total time to complete the task was the variable which distinguished the two groups the most. It is difficult to relate these findings to previous studies. First, Rempfer et al. (2003) did not include a control group, so a direct comparison with this study is not possible. Second, and in part due to the fact that our shopping task was computerized (allowing a large number of variables to be calculated in a quantitative and precise manner), we included a large number of (relatively specific) variables. In contrast, Semkovska et al. (2004) only considered three (relatively broad) variables in their shopping task: sequencing errors, omissions, and repetitions. They found that patient and control groups differed significantly for number of sequencing errors and the repetitions variable. Repetitions may be considered similar to the aisle redundancy variable used in the present study, which also significantly distinguished between the patient and control groups. The sequencing errors variable is difficult to relate to the present study as this variable has a more global and composite nature and, therefore, 
Table 4. Correlations between cognitive variables and performance on the computerized shopping task in the patient group

\begin{tabular}{|c|c|c|c|c|c|c|}
\hline & Total time & $\begin{array}{l}\text { Number of } \\
\text { correct articles }\end{array}$ & $\begin{array}{c}\text { Aisle } \\
\text { redundancy }\end{array}$ & $\begin{array}{c}\text { Number of times a } \\
\text { non-pertinent aisle } \\
\text { was visualized }\end{array}$ & $\begin{array}{c}\text { Number of times } \\
\text { consult list }\end{array}$ & $\begin{array}{c}\text { Time spent } \\
\text { consulting } \\
\text { the list }\end{array}$ \\
\hline $\begin{array}{l}\text { Pre-morbid IQ } \\
\quad(\text { NART })\end{array}$ & 0.06 & 0.24 & 0.02 & -0.03 & 0.10 & $0.35^{\mathrm{a}}$ \\
\hline $\begin{array}{l}\text { Global cognitive } \\
\text { test score }\end{array}$ & -0.27 & $0.42 *$ & -0.21 & 0.31 & $-0.34^{b}$ & -0.32 \\
\hline $\begin{array}{l}\text { Phonological } \\
\text { verbal fluency }\end{array}$ & -0.08 & 0.09 & 0.24 & -0.14 & 0.21 & -0.03 \\
\hline $\begin{array}{l}\text { Semantic verbal } \\
\text { fluency }\end{array}$ & -0.14 & $0.41 *$ & -0.05 & $-0.41 *$ & 0.19 & 0.15 \\
\hline $\begin{array}{l}\text { CVLT Total } \\
\quad \text { correct } 1-5\end{array}$ & 0.03 & $0.36^{*}$ & -0.05 & -0.19 & 0.18 & 0.04 \\
\hline TMT B-A (Time) & $0.47 * *$ & -0.26 & 0.21 & $0.36^{*}$ & -0.12 & 0.11 \\
\hline Zoo map & 0.12 & $0.39 *$ & -0.14 & -0.22 & 0.09 & 0.20 \\
\hline $\begin{array}{l}\text { Stroop Color } \\
\text { Naming Time }\end{array}$ & 0.15 & $-0.60 * * *$ & -0.09 & $0.36^{*}$ & $-0.52 * *$ & -0.23 \\
\hline $\begin{array}{l}\text { Stroop Time } \\
\text { interference }\end{array}$ & 0.28 & $-0.42 *$ & -0.17 & 0.23 & -0.29 & -0.16 \\
\hline $\begin{array}{l}\text { WAIS Processing } \\
\text { speed index }\end{array}$ & -0.27 & $0.39 *$ & -0.10 & -0.18 & 0.22 & -0.02 \\
\hline $\begin{array}{c}\text { Letter-number } \\
\text { sequencing }\end{array}$ & 0.01 & 0.05 & 0.16 & 0.04 & 0.13 & -0.16 \\
\hline GAF & 0.03 & 0.28 & $0.54 * *$ & 0.02 & $0.41 *$ & 0.22 \\
\hline PANSS Total & 0.09 & $-0.51 *$ & -0.31 & 0.19 & $-0.59 * *$ & -0.31 \\
\hline Positive sub-scale & 0.16 & $-0.45^{*}$ & -0.09 & 0.20 & $-0.42 *$ & -0.24 \\
\hline Negative sub-scale & 0.18 & $-0.46^{*}$ & $-0.46^{*}$ & 0.20 & $-0.59 * *$ & -0.19 \\
\hline $\begin{array}{l}\text { General } \\
\text { psychopathology } \\
\text { sub-scale }\end{array}$ & -0.13 & $-0.46^{*}$ & -0.18 & 0.17 & $-0.49 *$ & -0.32 \\
\hline
\end{tabular}

Note . NART $=$ National Adult Reading Test CVLT $=$ California Verbal Learning Test $;$ TMT $=$ Trail Making Test; WAIS $=$ Wechsler Adult Intelligence Scale; GAF = Global Assessment of Functioning Scale; PANSS = Positive and Negative Syndrome Scale.

aFor the correlation between Time spent consulting the list and Pre-morbid IQ, there was a statistical tendency $(p=.059)$.

${ }^{\mathrm{b}}$ For the correlation between Number of times consult list and Global cognitive test score, there was a statistical tendency $(p=.069)$.

$* p<.05 ; * * p<.01 ; * * * p<.001$.

includes several of the variables included in the present study such as aisle redundancy and number of times a nonpertinent aisle was visualized. Nevertheless, it is interesting to note that both of these variables distinguished the two groups in the present study.

Relations between cognitive tests and the computerized shopping task in the patient group were examined. One shopping task variable (number of correct articles) significantly correlated with the global cognitive score. Furthermore, when correlations between specific cognitive tests and performance on the shopping task were carried out, this revealed significant relations with cognitive flexibility, verbal episodic memory, planning, processing speed, and inhibition. In Rempfer et al. (2003), accuracy (whether participants selected the correct item, at the correct size, and at the lowest price) was related to verbal memory, processing speed, and executive functioning. This is highly similar to findings from the present study, where total number of correct articles was also significantly related to verbal memory, processing speed and executive functioning. Semkovska et al. (2004) observed that sequencing errors (e.g., arriving at the cash register and then returning to get a missing item) was the only variable in their shopping task that significantly correlated with a cognitive variable (Picture Arrangement subtest of the WAIS-R). In the present study, a variable that tapped into similar errors (number of times a non-pertinent aisle was visualized) was associated with cognitive flexibility and semantic fluency.

A general pattern in the results was that a majority (four of six) of the variables of the computerized shopping task were associated with either cognitive flexibility or processing speed, or with both. Processing speed was significantly correlated with the number of correct articles (Stroop Color Naming time, WAIS Processing speed index), the number of times a non-pertinent aisle was visualized (Stroop Color Naming time), and the number of times participants consulted the shopping list (Stroop Color Naming time). This finding suggests that processing speed is closely related to level of functioning, as measured by the computerized shopping task. Similar findings were reported in Rempfer et al. (2003) where processing speed was related to performance 
on several measures of their shopping task. Important to note is that Semkovska et al. (2004) did not include a processing speed measure in their study. Recent years has revealed the importance of processing speed in schizophrenia. Dickinson et al. (2007) have argued that slowed information processing is a central feature of the cognitive impairment in schizophrenia as it: reveals large effect sizes (even relative to other central cognitive domains in schizophrenia such as verbal memory, executive functioning, and working memory), is reliable, is more discriminating of diagnostic group status than other cognitive domains, and is intimately related to prognosis and (social and professional) functional outcome in schizophrenia. Furthermore, these large effect sizes for processing speed were not sensitive to medication status (neither a contrast of medicated and medication-free samples, nor a contrast of low versus high dose antipsychotic treatment samples, produced significant differences).

Cognitive flexibility was also associated with performance on the shopping task in patients diagnosed with schizophrenia. This included the total time to complete the shopping task (TMT B-A), the number of correct articles chosen (semantic verbal fluency), and the number of times a non-pertinent aisle was visualized (semantic verbal fluency, TMT B-A). Performance on the Trail Making Task (Part B) is predominantly associated with cognitive flexibility (Kortte, Horner, \& Windham, 2002). In this context, according to their gateway hypothesis, Burgess, Dumontheil, and Gilbert (2007) have proposed that one important class of situation (primarily regulated by the rostral prefrontal cortex) is one where individuals find themselves in an open-ended, multitasking situation that requires the continuous shifting between internal (self-generated and maintained representations or goals) and external (stimulus-oriented attending) modes. This situation is not unlike the one participants found themselves in when performing the computerized shopping task: a novel situation was presented to them, they were required to create a plan of action (self-developed goals), to maintain this plan of action throughout the task, and to continuously shift between internal (self-developed and maintained goals) and external (task-related stimuli presented on the computer screen) modes. Semkovska et al. (2004) also reported significant correlations between their measure of cognitive flexibility (the flexibility subtask of the modified Stroop Color Word Test) and performance on the everyday living task (albeit only for choosing the menu and cooking the meal tasks and not for the shopping task), as did Rempfer et al. (2003) between redundancy scores on their shopping task and scores on the verbal fluency task.

Another general pattern was that the number of correct articles was correlated with several cognitive variables, whereas the other variables of the computerized shopping task were related to more specific cognitive variables. This suggests that the task of finding the correct articles is a highly complex and multifaceted task. In particular, the number of correct articles was significantly associated with verbal episodic memory, planning, processing speed, inhibition, cognitive flexibility, and semantic fluency. Similarly, Rempfer et al. (2003) re- ported their accuracy variable (the extent to which participants select the correct item at the correct size and at the lowest price for each of the 10 items) to be significantly associated with executive functioning (number of perseverative errors on the WCST), verbal memory, and processing speed.

The fact that other variables of the shopping task were associated with more specific cognitive variables in the present study is also similar to previous studies. Total time in the present study and in Rempfer et al. (2003) was only associated with one cognitive variable, although this variable differed between studies (cognitive flexibility in the present study and verbal memory in Rempfer et al. (2003). Again, it is difficult to compare findings with Semkovska et al. (2004) as only one association was found between the cognitive variables (Picture Arrangement) and performance on the shopping task (sequencing errors).

It makes sense that performance on the computerized shopping task was related to episodic memory, cognitive flexibility, planning, and inhibition. Effective shopping behavior requires the involvement of all of these cognitive functions: a novel situation was presented to participants, who were then required to create a plan of action (planning), to maintain this plan in mind throughout the task (memory), to inhibit irrelevant stimuli during the task (inhibition), and to continuously shift between internal (internal thoughts) and external (stimuli presented to the participant by the computer screen) modes (cognitive flexibility). Planning was related to the number of correct articles chosen in the present study, and indeed planning is an important function as it allows one to efficiently explore the grocery store in an organized manner. Number of correct articles was correlated with verbal episodic memory. Clearly, verbal episodic memory is involved in trying to remember the articles that need to be chosen. Inhibition is essential so as to inhibit irrelevant stimuli while performing the task. In the present study, inhibition was particularly associated with the number of correct articles. Finally, cognitive flexibility is involved in shopping behavior as one is required to continuously shift between internal (self-generated and maintained representations or goals) and external (stimulus-oriented attending) modes, and which is typically involved in multitasking and "ill-structured" situations (Burgess et al., 2007).

Performance on the computerized shopping task was significantly associated with several demographic and clinical variables, and with social functioning. In contrast, only one cognitive measure (inhibition) correlated significantly with social functioning. Furthermore, none of the cognitive variables correlated significantly with the other clinical variables. Only processing speed and inhibition significantly differentiated between hospitalized and non-hospitalized patients. This suggests that the computerized shopping task is tapping into social functioning and clinical status of patients to a greater extent than the standard cognitive tests, with the exception of inhibition and processing speed. These findings are difficult to relate to previous studies, as a global social functioning measure was not included in both Rempfer et al. (2003) and Semkovska et al. (2004). 
It is worth mentioning the fact that, when patients were divided into those who had successfully collected all seven items and those who did not, this did not reveal any significant group differences for both clinical and cognitive variables. Thus, although being able to collect all pertinent items when shopping is a clinically pertinent variable, it was not found to discriminate on a cognitive and clinical level, suggesting that this variable might depend on other factors.

Both number of correct articles and the number of times participants consulted the list were related to symptomatology, whereas other shopping task variables were largely unrelated to severity of symptoms. Number of correct articles may be considered the best measure of task success, and this variable was, furthermore, positively related to most cognitive variables and negatively related to symptom ratings. That is, less number of correct articles was related to both poorer performance on the cognitive tests and increased symptom severity. Of interest, however, the number of times participants consulted the list was negatively related to symptomatology (i.e., less severe symptomatology was related to frequent consultations of the list) and positively correlated with the global social functioning measure (i.e., better social functioning was related to frequent consultations of the list). This pattern of results was also the case for aisle redundancy for social functioning (i.e., better social functioning was related to increased aisle redundancy) and for symptoms - albeit only for negative symptoms (i.e., increased aisle redundancy was related to less severe negative symptoms). This suggests that being careful and doublechecking on the shopping task may in fact be useful, or at least associated with less severe symptomatology and more effective everyday functioning.

A limit of the present study is that only a general social functioning measure (GAF) was included and, therefore, future studies are required which include more comprehensive measures of social functioning. Such a study is currently under way. Important to note is that, although a computerized version of an everyday task (such as the one used in the present study) has several advantages (e.g., a great number of variables can be quantified with precision, etc.), there are differences with such a task and the same task in natural environments (i.e., actually shopping in a real grocery store). A necessary addition to the present study's methodology would be to administer both a real-life shopping task and the computerized shopping task to patients with schizophrenia to examine the external validity of the computerized shopping task. Finally, the present study is exploratory in nature and thus further replication is clearly needed which will take into account the above-mentioned limits and which will include larger samples of participants.

\section{ACKNOWLEDGMENTS}

This study was supported in part by an unrestricted grant from AstraZeneca Belgium. The authors thank the Informatics Department of the Intercommunale de Soins Spécialisés de Liège (Mental Health Sector) and, in particular, Steve Lanuit for his help in programming the learning phase and the shopping task. Authors declare that they have no conflict of interest.

\section{REFERENCES}

American Psychiatric Association. (1994). Diagnostic and Statistical Manual of Mental Disorders (4th ed.). Washington, DC: American Psychiatric Association Press.

Army Individual Test Battery. (1944). Manual of Directions and Scoring. Washington, DC: War Department, Adjutant General's Office.

Bowie, C.R., Reichenberg, A., Patterson, T.L., Heaton, R.K., \& Harvey, P.D. (2006). Determinants of real-world functioning performance in schizophrenia: Correlations with cognition, functional capacity, and symptoms. American Journal of Psychiatry, 163, 418-425.

Brickenkamp, R. (1966). Le Test d2 d'attention concentrée. Paris: Editest.

Burgess, P.W., Dumontheil, I., \& Gilbert, S.J. (2007). The gateway hypothesis of rostral prefrontal cortex (area 10) function. Trends in Cognitive Sciences, 11, 290-298.

Cardebat, D., Doyon, B., Puel, M., Goulet, P., \& Joannette, Y. (1990). Evocation lexicale formelle et sémantique chez des sujets normaux: Performances et dynamiques de production en fonction du sexe, de l'âge et du niveau d'études. Acta Neurologica Belgica, 90, 207-217.

Delis, D., Freeland, J., Kramer, J., \& Kaplan, E. (1988). Integrating clinical assessment with cognitive neuroscience: Construct validation of California Verbal Learning Test. Journal of Consulting and Clinical Psychology, 56, 123-130.

Dickinson, D., Ramsey, M.E., \& Gold, J.M. (2007). Overlooking the obvious: A meta-analytic comparison of Digit Symbol Coding tasks and other cognitive measures in schizophrenia. Archives of General Psychiatry, 64, 532-542.

Fioravanti, M., Carlone, O., Vitale, B., Cinti, M.E., \& Clare, L. (2005). A meta-analysis of cognitive deficits in adults with a diagnosis of schizophrenia. Neuropsychology Review, 15, 73-95.

Golden, C.J. (1978). Stroop Color and Word Test: A Manual for Clinical and Experimental Uses. Chicago: Skoelting.

Green, M.F. (1996). What are the functional consequences of neurocognitive deficits in schizophrenia? American Journal of Psychiatry, 153, 321-330.

Green, M.F., Kern, R.S., Braff, D.L., \& Mintz, J. (2000). Neurocognitive deficits and functional outcome in schizophrenia: Are we measuring the right stuff? Schizophrenia Bulletin, 26, 119-136.

Hamera, E., \& Brown, C. (2000). Developing context-based performance measures: Grocery shopping skills in individuals with schizophrenia. American Journal of Occupational Therapy, 54, 20-25.

Hamera, E., Brown, C., Rempfer, M., \& Davis, N. (2002). Test of grocery shopping skills: Discrimination of people with and without mental illness. Psychiatric Rehabilitation Skills, 6, 296-311.

Heaton, R.K., Chelune, G., Talley, J.L., Kay, G.G., \& Curtiss, G. (1993). Wisconsin Card Sorting Test Manual. Odessa, FL: Psychological Assessment Resources.

Heinrichs, R.W., \& Zakzanis, K.K., (1998). Neurocognitive deficit in schizophrenia: A quantitative review of the evidence. Neuropsychology, 12, 426-445.

Hilsenroth, M.J., Ackerman, S.J., Blagys, M.D., Baumann, B.D., Baity, M.R., Smith, S.R., et al. (2000). Reliability and validity of DSM-IV axis V. American Journal of Psychiatry, 157, 1858-1863. 
Jones, S.H., Thornicroft, G., Coffey, M., \& Dunn, G. (1995). A brief mental health outcome scale: Reliability and validity of the Global Assessment of Functioning (GAF). British Journal of Psychiatry, 166, 654-659.

Kay, S.R., Opler, L.A., \& Fiszbein, A. (1987). Positive and Negative Syndrome Scale (PANSS) Rating Manual. San Rafael, CA: Social and Behavioral Sciences Documents.

Kern, R.S., Green, M.F., Mintz, J., \& Liberman, R.P. (2003). Does "Errorless Learning" compensate for neurocognitive impairments in the work rehabilitation of persons with schizophrenia? Psychological Medicine, 33, 433-442.

Kern, R.S., Liberman, R.P., Kopelowicz, A., Mintz, J., \& Green, M.F. (2002). Application of "Errorless Learning" for improving work performance in persons with schizophrenia. American Journal of Psychiatry, 159, 1921-1926.

Kortte, K.B., Horner, M.D., \& Windham, W.K. (2002). The Trail Making Test, Part B: Cognitive flexibility or ability to maintain set? Applied Neuropsychology, 9, 106-109.

Kurtz, M.M., Baker, E., Pearlson, G.D., \& Astur, R.S. (2007). A virtual reality apartment as a measure of medication management skills in patients with schizophrenia: A pilot study. Schizophrenia Bulletin, 33, 1162-1170.

Mackinnon, A., \& Mulligan, R. (2005). Estimation de l'intelligence prémorbide chez les francophones. L'Encéphale, 31, 31-43.

McGurk, S.R., \& Meltzer, H.Y. (2000). The role of cognition in vocational functioning in schizophrenia. Schizophrenia Research, 45, 175-185.

McKibbin, C.L., Brekke, J.S., Sires, D., Jeste, D.V., \& Patterson, T.L. (2004). Direct assessment of functional abilities: Relevance to persons with schizophrenia. Schizophrenia Research, 72, 53-67.

Nelson, H.E. (1982). National Adult Reading Test. Windsor: NFER-Nelson.

O'Carroll, R.E., Russell, H.H., Lawrie, S.M., \& Johnstone, E.C. (1999). Errorless learning and the cognitive rehabilitation of memory-impaired schizophrenic patients. Psychological Medicine, 29, 105-112.

Patterson, T.L., Goldman, S., McKibbin, C.L., Hughs, T., \& Jeste, D.V. (2001). UCSD Performance-based skills assessment: Development of a new measure of everyday functioning for severely mentally ill adults. Schizophrenia Bulletin, 27, 235-245.

Poitenaud, J., Deweer, B., Kalafat, M., \& Van der Linden, M. (2007). Adaptation en langue française du California Verbal Learning Test. Paris: Les Editions du Centre de Psychologie Appliquée.

Rempfer, M., Hamera, E., Brown, C., \& Cromwell, R. (2003). The relations between cognition and the independent living skill of shopping in people with schizophrenia. Psychiatry Research, 117, 103-112.

Semkovska, M., Bédard, M.A., Godbout, L., Limoge, F., \& Stip, E. (2004). Assessment of executive dysfunction during activities of daily living in schizophrenia. Schizophrenia Research, 69, 289-300.

Velligan, D.I., Bow-Thomas, C., Mahurin, R.K., Miller, A.L., \& Halgunseth, L.C. (2000). Do specific neurocognitive deficits predict specific domains of community functioning in schizophrenia? Journal of Nervous and Mental Disease, 188, 518-524.

Wechsler, D. (1981). Wechsler Adult Intelligence Scale-Revised Manual. New York: Psychological.

Wechsler, D. (2000). WAIS-III: Echelle d'Intelligence de Wechsler pour Adultes (3rd ed.). Paris: Les éditions du Centre de Psychologie Appliquée.

Wechsler, D. (2001). MEM-III: Manuel de l'Echelle Clinique de Mémoire (3rd ed.). Paris: Les éditions du Centre de Psychologie Appliquée.

Wilson, B.A., Alderman, N., Burgess, P.W., Emslie, H., \& Evans, J.J. (1996). BADS: Behavioural Assessment of the Dysexecutive Syndrome. Bury St Edmunds, UK: Thames Valley Test Company. 